\title{
Magnetic-vortex Dynamic Quasi-crystal Formation in Soft Magnetic Nano-disks
}

\author{
Junhoe Kim and Sang-Koog Kim* \\ National Creative Initiative Center for Spin Dynamics and Spin-Wave Devices, Nanospinics Laboratory, \\ Research Institute of Advanced Materials, Department of Materials Science and Engineering, Seoul National University, \\ Seoul 08826, Republic of Korea
}

(Received 22 February 2017, Received in final form 10 March 2017, Accepted 14 March 2017)

\begin{abstract}
We report a micromagnetic numerical study on different quasi-crystal formations of magnetic vortices in a rich variety of dynamic transient states in soft magnetic nano-disks. Only the application of spin-polarized dc currents to a single magnetic vortex leads to the formation of topological-soliton quasi-crystals composed of different configurations of skyrmions with positive and negative half-integer numbers (magnetic vortices and antivortices). Such topological object formations in soft magnets, not only in the absence of DzyaloshinskiiMoriya interaction but also without magnetocrystalline anisotropy, are discussed in terms of two different topological charges, the winding number and the skyrmion number. This work offers an insight into the dynamic topological-spin-texture quasi-crystal formations in soft magnets.
\end{abstract}

Keywords : topological soliton, half-integer skyrmion, vortex and antivortex, dynamic quasi-crystal

\section{Introduction}

Topologically non-trivial spin textures, such as the magnetic bubble domain [1, 2], the magnetic vortex [2-4], and antivortex [2] as well as the pairwise vortex and antivortex in confined magnetic elements [5-9], have attracted rapidly growing interest due to their fundamental dynamic characteristics [10-18] and potential applications to information-storage $[13,19,20]$ and -processing devices [2123]. The magnetic skyrmion [24-26], for example, represents peculiar spin configurations wherein spins point in all of the directions wrapping a three-dimensional (3D) sphere, thereby generating an integer Skyrmion topological charge [24, 25]. Magnetic skyrmions have been found in special magnetic materials of non-centro symmetry $[26,27]$ or in hybrid ultrathin magnetic films where the inversion symmetry is broken by the presence of heterointerfaces $[28,29]$. The non-centro symmetry, either in bulk or at the interface with strong spin-orbit coupling, causes the Dzyaloshinskii-Moriya (DM) interaction [30, 31 ] that plays a crucial role in Skyrmion formation in bulk and thin film [26-29]. From the application point of view, the nanoscale dimensions and low-power manipulation of

(C)The Korean Magnetics Society. All rights reserved.

*Corresponding author: Tel: +82-2-880-5854

Fax: +82-2-885-1457, e-mail: sangkoog@snu.ac.kr the magnetic skyrmions are very advantageous features [29, 32, 33].

On the other hand, the magnetic vortex has been intensively studied for its peculiar static and dynamic characteristics $[3,4,10,11,16-18]$. Its fine magnetic structure shows either counter-clockwise (CCW) or clockwise (CW) curling in-plane magnetization with either upward or downward out-of-plane magnetization at the core region in confined magnetic elements [2-4]. In the 3D topological aspect, the magnetic vortex has a half-integer skyrmion number, expressed as $q=n p / 2$ (Ref. 34) with the winding number of $n=+1$ and a given polarization $p$ $=+1(-1)$ for upward (downward) core magnetization. Also, the antivortex $(n=-1)$, as the topological counterpart of the vortex, is found in parts of cross-tie walls [5, $7,35]$ where vortices and antivortices are periodically alternating or in a metastable state in specially designed geometrical confinements. In ultrafast dynamic processes, the vortex and antivortex are created and annihilated to conserve the total winding number in a given system; thus always their creation and annihilation are pairwise [6-9]. In this article, we report our findings on the formation of dynamic quasi-crystals composed of positive and negative half-integer skyrmions (magnetic vortices and antivortices) in many different configurations in dynamic transient states in soft magnetic (e.g., Permalloy) disks without consideration of DM interaction and with zero magneto- 
crystalline anisotropy. The formation of such topologicalspin-texture quasi-crystals is addressed in terms of two different topological charges, $n$ and $q$.

\section{Methods}

In our micromagnetic numerical calculation, we employed the OOMMF code (version 1.2a4) [36] using the Landau-Lifshitz-Gilbert equation with Slonczewski spintransfer torque [37-39], expressed as $d \mathbf{M} / d t=-\gamma /(1+$ $\left.\alpha^{2}\right)\left(\mathbf{M} \times \mathbf{H}_{\mathrm{eff}}+\left(\alpha / M_{s}\right)\left(\mathbf{M} \times\left(\mathbf{M} \times \mathbf{H}_{\mathrm{eff}}\right)\right)\right)+\left(a_{\mathrm{STT}} / M_{s}\right) \mathbf{M} \times(\mathbf{M}$ $\left.\times \hat{\mathbf{m}}_{p}\right)$ with $a_{\mathrm{STT}}=\frac{1}{2 \pi} h \gamma P j_{0} /\left(\mu_{0} 2 e M_{s} L\right)$, where $\alpha$ is the damping constant, $\gamma$ the gyromagnetic ratio, $\mathbf{H}_{\text {eff }}$ the effective field, $h$ the Planck's constant, $j_{0}$ the current density, $\mu_{0}$ the vacuum permeability, $e$ the electron charge, $M_{\mathrm{s}}$ the saturation magnetization, $P$ the degree of spin polarization, and $\hat{\mathbf{m}}_{p}$ the unit vector of the spin-polarization direction. The well-known Py material parameters were applied: $M_{S}$ $=8.6 \times 10^{5} \mathrm{~A} / \mathrm{m}$, exchange stiffness $A_{e x}=1.3 \times 10^{-11} \mathrm{~J} / \mathrm{m}$, $\alpha=0.01$, and with zero magnetocrystalline anisotropy. The unit cell size was $2 \times 2 \times 10 \mathrm{~nm}^{3}$.

\section{Results and Discussion}

In the present study, a model system comprising a freestanding Permalloy $\left(\mathrm{Py}: \mathrm{Ni}_{80} \mathrm{Fe}_{20}\right)$ nano-disk of radius $R=$ $150 \mathrm{~nm}$ and thickness $L=10 \mathrm{~nm}$ was used (see Fig. 1). The initial ground state shows a single vortex of upward core magnetization $(p=+1)$ and clockwise $(\mathrm{CW})$ in-plane curling magnetization (chirality $c=-1$ ). Out-of-plane dc currents (denoted as $i_{\mathrm{p}}=+1$ and $m_{\mathrm{p}}=-1$ ) of densities (a)

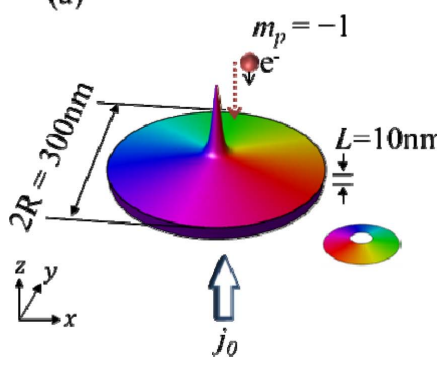

(b)

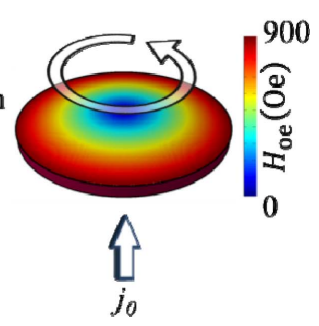

Fig. 1. (Color online) (a) Model of free-standing Py disk with magnetic vortex structure in initial ground state. The color indicates the local in-plane curling magnetizations, and the height of the surface corresponds to the out-of-plane magnetizations. The direction of the out-of-plane current is denoted as $i_{\mathrm{p}}=+1$, and the spin polarization is denoted as $m_{\mathrm{p}}=-1$. (b) Spatial distribution of circumferential OHs induced by current flow of density $j_{0}=1.0 \times 10^{8} \mathrm{~A} / \mathrm{cm}^{2}$. The colors and arrows represent the strength and rotation sense of the OHs, respectively.

varying within the $1.0 \sim 2.0 \times 10^{8} \mathrm{~A} / \mathrm{cm}^{2}$ range and a spin polarization of $P=0.7$ were applied in the $+\hat{\mathbf{z}}$ direction. The circumferential Oersted fields (OHs) due to the current flow were obtained from the Biot-Savart's formulation [40] (see Fig. 1(b)). Note that the field direction and the in-plane curling magnetization in the initial ground state are antiparallel. The local current densities through the entire disk were assumed to be uniform.

As reported earlier [15, 40-43], application of an out-ofplane dc current with a high current density, here $j_{0}=1.0$ $\times 10^{8} \mathrm{~A} / \mathrm{cm}^{2}$, leads to overall vortex-core switching from the upward to downward core orientation along with

(a) position (nm) position (nm)

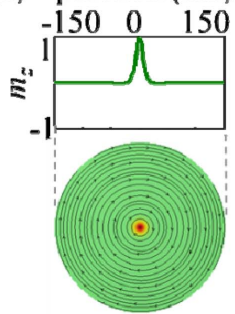

(b)

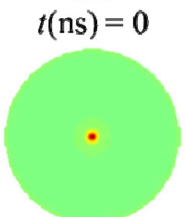

$Q=[0.4864]$

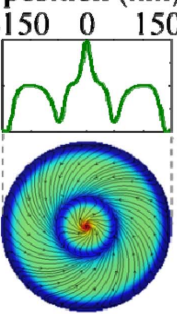

0.118

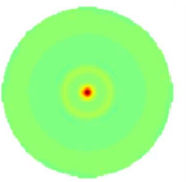

[0.9708]

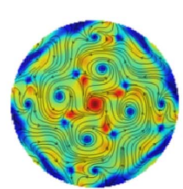

0.308

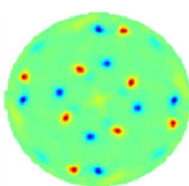

[1.802]

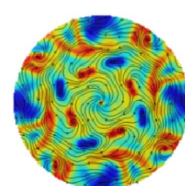

0.471

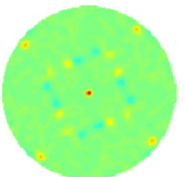

[1.223]

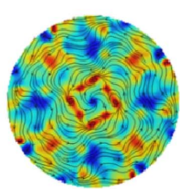

0.580

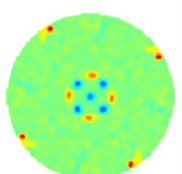

[1.434]

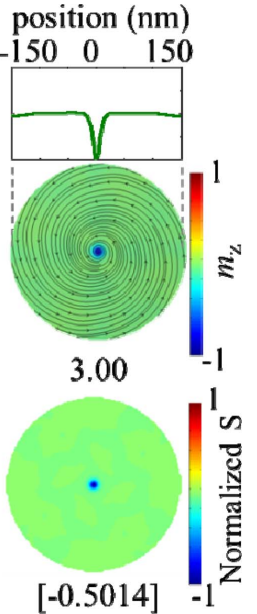

$[-0.5014]-1$

Fig. 2. (Color online) Snapshot plane-view images of spatial distribution of (a) local magnetizations and (b) corresponding skyrmion densities normalized to maximum value at indicated time. In (a), the streamlines with small arrows indicate the local inplane magnetizations, and the colors correspond to the out-of-plane magnetizations normalized by the saturation magnetization, $m_{\mathrm{z}}$. The insets show the $m_{\mathrm{z}}$ profiles across the center position of a given disk. 
CCW-to-CW chirality switching (compare the two images taken at $t=0$ and $t=3.0$ ns shown in Fig. 2(a)). According to the current density, a rich variety of dynamic behaviors have been found (Refs. 15, 40, 44, and 45). Here, we focus on some of the fine dynamic processes driven by such high current densities. Figure 2(a) shows additional transient states towards the vortex structure with the $\mathrm{CW}$ chirality and the downward polarization. A bubble-domain state appears at $t=0.118 \mathrm{~ns}$, as evidenced by the $m_{\mathrm{z}}\left(=\mathbf{M} \cdot \hat{z} / M_{s}\right)$ profile across the disk center (see the inset of Fig. 2(a)). The $m_{z}$ profile clearly shows, as representative of that domain, the unique variation of $m_{z}$ $\sim+1$ at the center and $m_{\mathrm{z}} \sim-1$ at the two outer regions. Additionally, other peculiar dynamic transient states appear serially at $t=0.308,0.471$, and 0.580 in different pairwise vortex-antivortex configurations. These transformations take place through dynamic subprocesses of the creation and annihilation of vortex-antivortex pairs in steps of a few tens of pico-seconds

In order to interpret those spin textures in terms of the creation and annihilation of 3D topological solitons, we numerically calculated the local skyrmion density $S=$ $\frac{1}{4 \pi} \boldsymbol{m} \cdot(\partial \boldsymbol{m} / \partial x \times \partial \boldsymbol{m} / \partial y)[26,46]$ and the total skyrmion number over the entire disk $Q=\iint S d x d y$. Figure 2(b) displays the resultant skyrmion densities normalized to the maximum values at the indicated times. Figure 2(b) clearly shows the many blue and red spots and their characteristic arrangements, representing highly localized solitons with the opposite half-integer skyrmions. The vortices and antivortices with either the up or down core orientation show themselves in the specific quasi-crystal arrangements.

Figure 3(a) illustrates the details of the three representative snapshot images taken at $t=0.308,0.471$, and $0.580 \mathrm{~ns}$, presenting the distribution of the winding numbers of the local topological solitons indexed in the following: vortices and antivortices correspond to $n=+1$ and $n=-1$, while the edge solitons near the disk boundary correspond to either $n=+1 / 2$ or $-1 / 2$. The winding number of the upward-core vortex in the initial state is $n=+1$, and is conserved during all of the transformations of the individual transient states. Compared with the winding-number representation, the corresponding skyrmion numbers of the local solitons, $q=n p / 2$, also are marked on the three representative snapshot images of the local skyrmion density distributions, as shown in Fig. 3(b). The total skyrmion number $Q=+1 / 2$ at the initial vortex with the up core orientation $(p=+1)$ varies, and turns out to be $Q$ $=+1$ for the bubble domain at $t=0.118 \mathrm{~ns}$ (see Fig. 2(b)). The $Q=+1$ skyrmion state immediately transforms into a (a) Winding number

(b) Skyrmion number

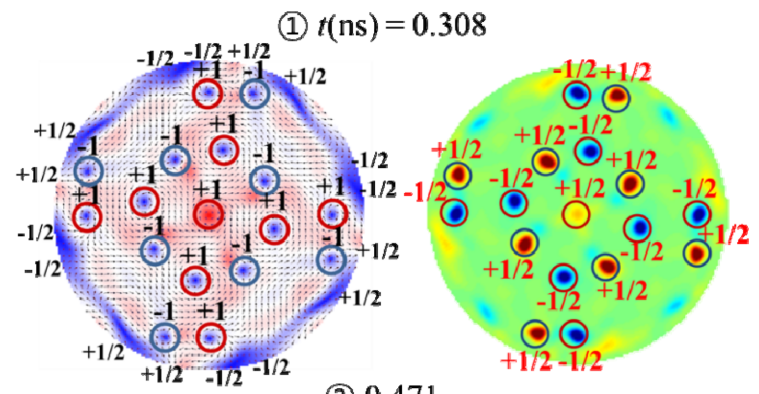

(2) 0.471
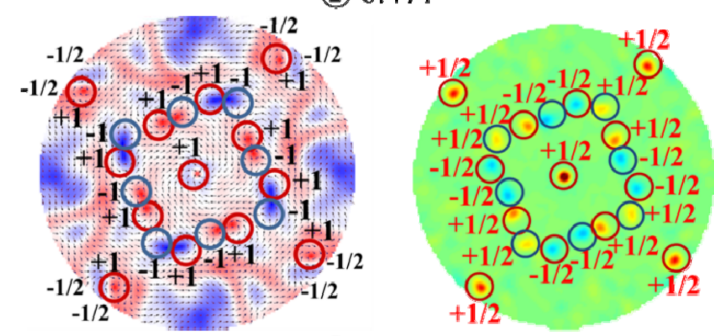

(3) 0.580
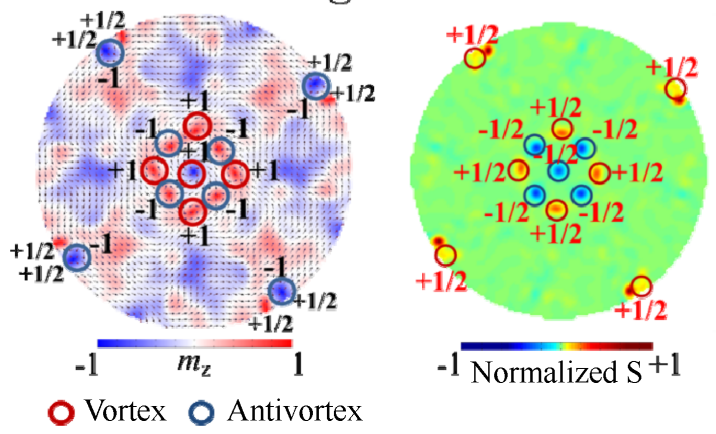

Fig. 3. (Color online) (a) Snapshot images of local magnetization distributions in whole disk area at indicated times $t=$ $0.308,0.471$, and $0.580 \mathrm{~ns}$, where vortices and antivortices are denoted as $n=+1$ and $n=-1$, respectively, and edge solitons are represented by either $n=+1 / 2$ or $n=-1 / 2$. (b) Corresponding skyrmion densities normalized to the given maximum value. The blue and red spots thus correspond to positive $(q=+1 / 2)$ and negative $(q=-1 / 2)$ half-integer skyrmions (magnetic vortices and antivortices), respectively.

rich variety of soliton-lattice quasi-crystal transient states as shown in Fig. 3(b). The representative skyrmion density images reveal that the vortices and the counterpart antivortices are created and annihilated in the pairwise fashion. For the same polarization in the vortex-antivortex pairs, the skyrmion number is always $q=+1 / 2$ in one and $q=$ $-1 / 2$ in the other, because the vortex and antivortex have the opposite winding-number signs (vortex: $n=+1$; antivortex: $n=-1$ ) and, thus, opposite half-integer skyrmion numbers. The blue and red spots in Fig. 3(b) indicate the positive and negative $q$ values, $+1 / 2$ and $-1 / 2$, respectively. The vortex and antivortex with the same polarization are determined simply by the sign of the local skrymion 
density value. In the course of the dynamic transformations, the creation and annihilation of vortex and antivortex is always pairwise so as to hold the zero winding number via topological winding-number conservation; and since each of the vortex-antivortex pairs has the same polarization here, the sum of the skyrmion numbers of each vortex-antivortex pair is always zero. While the total winding number over the disk is maintained as $n=+1$ (vortex), the total skyrmion number $Q$ is not conserved but varies during the dynamic transformations, as evidenced by the $Q$ variation shown in Fig. 2(b). The processes of the creation and annihilation of vortex-antivortex pairs correspond to the topological sector as uniform ground states, without any spin-wave emission, as addressed in Refs. [9, 34].

\section{Conclusions}

In summary, we studied formations of an integer skyrmion bubble domain and of positive and negative half-integer skyrmions (magnetic vortices and antivortices) in a rich variety of dynamic quasi-crystal arrangements during the dynamic process of a single vortex state to another vortex state in a soft magnetic disk of non-magnetocrystalline anisotropy and non-DM interaction. Those dynamic transformations occur while conserving the total winding number in a given system and maintaining the same topological sector from the topological charge aspect. This work provides a fundamental understanding of the dynamic quasi-crystal formations of topological spin textures such as vortices and antivortices in geometrically confined soft magnets.

\section{Acknowledgements}

This research was supported by the Basic Science Research Program through the National Research Foundation of Korea (NRF) funded by the Ministry of Science, ICT \& Future Planning (NRF-2015R1A2A1A10056286).

\section{References}

[1] A. Malozemoff and J. Slonzewski, Magnetic Domain Walls in Bubble Materials, Academic, New York (1979).

[2] A. Hubert and R. Schäfer, Magnetic Domains: The Analysis of Magnetic Microstructure, Springer, Berlin (1998).

[3] T. Shinjo, T. Okuno, R. Hassdorf, K. Shigeto, and T. Ono, Science 289, 930 (2000).

[4] A. Wachowiak, J. Wiebe, M. Bode, O. Pietzsch, M. Morgenstern, and R. Wiesendanger, Science 298, 577 (2002).

[5] K. Shigeto, T. Okuno, K. Mibu, T. Shinjo, and T. Ono, Appl. Phys. Lett. 80, 4190 (2002).
[6] K.-S. Lee, B.-W. Kang, Y.-S. Yu, and S.-K. Kim, Appl. Phys. Lett. 85, 1568 (2004).

[7] K.-S. Lee, S. Choi, and S.-K. Kim, Appl. Phys. Lett. 87, 192502 (2005).

[8] B. Van Waeyenberge, A. Puzic, H. Stoll, K. W. Chou, T. Tyliszczak, R. Hertel, M. Fähnle, H. Brückl, K. Rott, G. Reiss, I. Neudecker, D. Weiss, C. H. Back, and G. Schütz, Nature 444, 461 (2006).

[9] R. Hertel and C. Schneider, Phys. Rev. Lett. 97, 177202 (2006).

[10] K. Y. Guslienko, B. A. Ivanov, V. Novosad, Y. Otani, H. Shima, and K. Fukamichi, J. Appl. Phys. 91, 8037 (2002).

[11] M. Buess, R. Höllinger, T. Haug, K. Perzlmaier, U. Krey, D. Pescia, M. R. Scheinfein, D. Weiss, and C. H. Back, Phys. Rev. Lett. 93, 077207 (2004).

[12] H. Wang and C. E. Campbell, Phys. Rev. B 76, 220407(R) (2007).

[13] R. P. Cowburn, Nature Mater. 6, 255 (2007).

[14] J. Thomas, Nature Nanotechnol. 2, 206 (2007).

[15] K.-S. Lee, M.-W. Yoo, Y.-S. Choi, and S.-K. Kim, Phys. Rev. Lett. 106, 147201 (2011).

[16] M. Kammerer, M. Weigand, M. Curcic, M. Noske, M. Sproll, A. Vansteenkiste, B. Van Waeyenberge, H. Stoll, G. Woltersdorf, C. H. Back, and G. Schuetz, Nature Commun. 2, 279 (2011).

[17] R. Wang and X. Dong, Appl. Phys. Lett. 100, 082402 (2012).

[18] M.-W. Yoo, J. Lee, and S.-K. Kim, Appl. Phys. Lett. 100, 172413 (2012).

[19] S.-K. Kim, K.-S. Lee, Y.-S. Yu, and Y.-S. Choi, Appl. Phys. Lett. 92, 022509 (2008).

[20] S. Bohlens, B. Krüger, A. Drews, M. Bolte, G. Meier, and D. Pfannkuche, Appl. Phys. Lett. 93, 142508 (2008).

[21] S. Barman, A. Barman, and Y. Otani, IEEE Trans. Magn. 46, 1342 (2010).

[22] H. Jung, Y.-S. Choi, K.-S. Lee, D.-S. Han, Y.-S. Yu, M.Y. Im, P. Fischer, and S.-K. Kim, ACS Nano 6, 3712 (2012).

[23] D.-S. Han, A. Vogel, H. Jung, K.-S. Lee, M. Weigand, H. Stoll, G. Schütz, P. Fischer, G. Meier, and S.-K. Kim, Sci. Rep. 3, 2262 (2013).

[24] T. H. R. Skyrme, Nucl. Phys. 31, 556 (1962).

[25] A. A. Belavin and A. M. Polyakov, JETP Lett. 22, 245 (1975).

[26] S. Mühlbauer, B. Binz, F. Jonietz, C. Pfleiderer, A. Rosch, A. Neubauer, R. Georgii, and P. Böni, Science 323, 915 (2009)

[27] X. Z. Yu, Y. Onose, N. Kanazawa, J. H. Park, J. H. Han, Y. Matsui, N. Nagaosa, and Y. Tokura, Nature 465, 901 (2010).

[28] S. Heinze, K. V. Bergmann, M. Menzel, J. Brede, A. Kubetzka, R. Wiesendager, G. Bihlmayer, and S. Blügel, Nature Physics 7, 713 (2011).

[29] A. Fert, V. Cros, and J. Sampaio, Nature Nanotech. 8, 152 (2013). 
[30] I. E. Dzyaloshinskii, J. Phys. Chem. Sol. 4, 241 (1958).

[31] T. Moriya, Phys. Rev. 120, 91 (1960).

[32] X. Z. Yu, N. Kanazawa, W. Z. Zhang, T. Nagai, K. Kimoto, Y. Matsui, Y. Onose, and Y. Tokura, Nature Commun. 3, 988 (2012).

[33] J. Iwasaki, M. Mochizuki, and N. Nagaosa, Nature Nanotech. 8, 742 (2013).

[34] O. Tretiakov and O. Tchernyshyov, Phys. Rev. B 75, 012408 (2007).

[35] E. E. Huber Jr., D. O. Smith, and J. B. Goodenough, J. Appl. Phys. 29, 294 (1958).

[36] The version of the OOMMF code used is 1.2a4. See http://math.nist.gov/oommf.

[37] L. D. Landau and E. M. Lifshitz, Phys. Z. Sowjetunion 8, 153 (1935).
[38] T. L. Gilbert, Phys. Rev. 100, 1243 (1955).

[39] J. C. Slonczewski, J. Magn. Magn. Mater. 159, L1 (1996).

[40] Y.-S. Choi, M.-W. Yoo, K.-S. Lee, Y.-S. Yu, H. Jung, and S.-K. Kim, Appl. Phys. Lett. 96, 072507 (2010).

[41] J.-G. Caputo, Y. Gaididei, F. G. Mertens, and D. D. Sheka, Phys. Rev. Lett. 98, 056604 (2007).

[42] D. D. Sheka, Y. Gaididei, and F. G. Mertens, Appl. Phys. Lett. 91, 082509 (2007).

[43] Y. Liu, H. He, and Z. Zhang, Appl. Phys. Lett. 91, 242501 (2007).

[44] O. M. Volkov, V. P. Kravchuk, D. D. Sheka, and Y. Gaididei, Phys. Rev. Lett. 84, 052404 (2011).

[45] Y. Gaididei, O. M. Volkov, V. P. Kravchuk, and D. D. Sheka, Phys. Rev. B 86, 144401 (2012).

[46] B. Binz, A. Vishwanath, Physica B 403, 1336 (2008). 